Provided for non-commercial research and education use. Not for reproduction, distribution or commercial use.

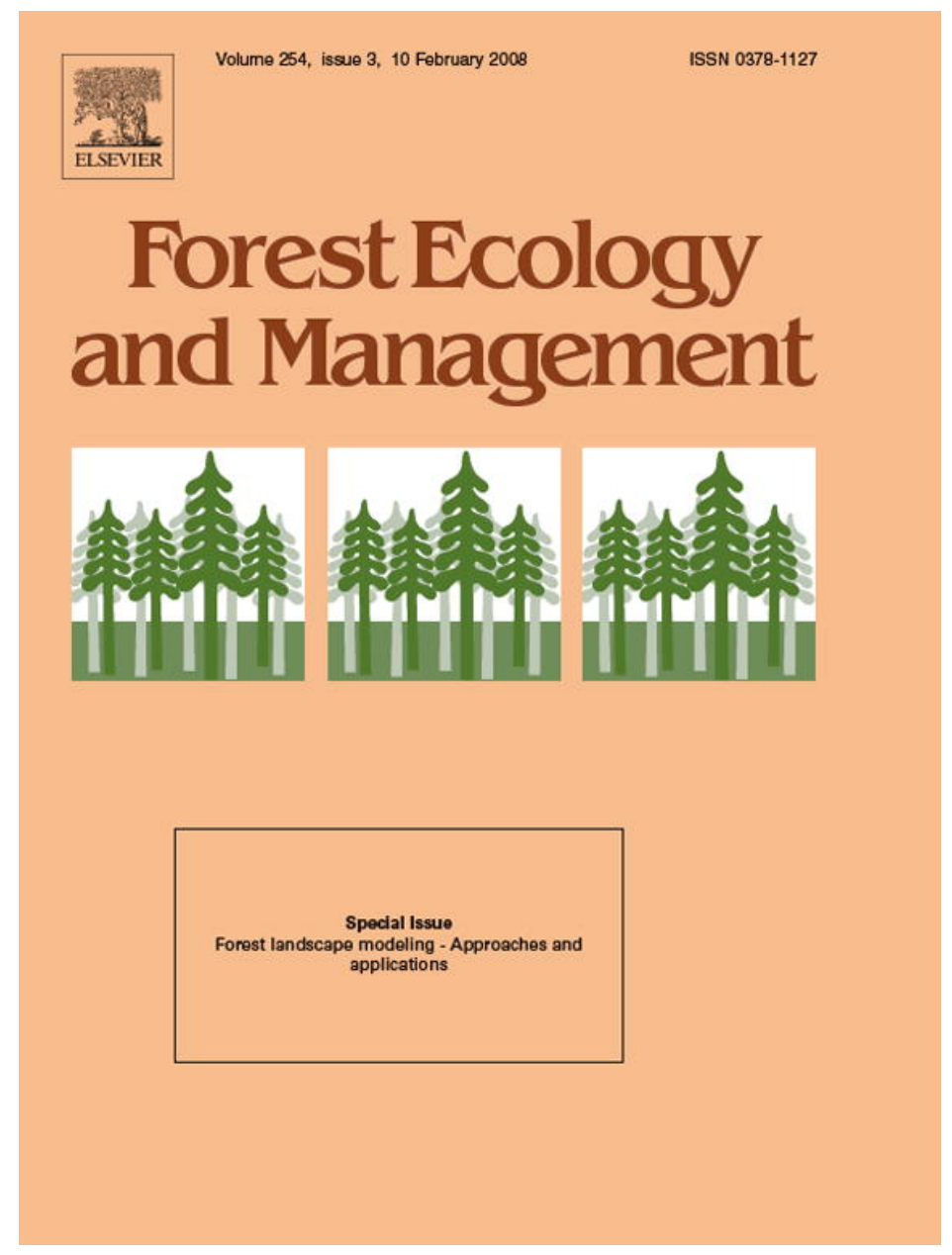

This article was published in an Elsevier journal. The attached copy

is furnished to the author for non-commercial research and education use, including for instruction at the author's institution, sharing with colleagues and providing to institution administration.

Other uses, including reproduction and distribution, or selling or licensing copies, or posting to personal, institutional or third party websites are prohibited.

In most cases authors are permitted to post their version of the article (e.g. in Word or Tex form) to their personal website or institutional repository. Authors requiring further information regarding Elsevier's archiving and manuscript policies are encouraged to visit:

http://www.elsevier.com/copyright 


\title{
A GIS based spatially explicit model of dispersal agent behavior
}

\author{
Fang Qiu ${ }^{\mathrm{a}, *}$, Bin Li ${ }^{\mathrm{b}}$, Bryan Chastain ${ }^{\mathrm{a}}$, Mohammed Alfarhan ${ }^{\mathrm{a}}$ \\ ${ }^{\text {a }}$ Program in Geographic Information Sciences, University of Texas at Dallas, P.O. Box 830688 GR31, Richardson, TX 75083, United States \\ ${ }^{\mathrm{b}}$ Department of Geography, Central Michigan University, Mount Pleasant, MI 48858, United States \\ Received 20 February 2007; received in revised form 24 May 2007; accepted 18 June 2007
}

\begin{abstract}
Spatial simulation models of seed dispersal have been constructed at the landscape level under the assumption of ubiquitous or uniform dispersibility. The anisotropic nature of vegetation distribution caused by different dispersal agents such as wind, gravity, water and animals were ignored. We propose a prototype of a GIS-based spatially explicit model of dispersal agent behavior (SEMODAR) to simulate the seed dispersal process by considering the unique behavioral characteristics of each seed dispersal agent. As a result, the influence of dispersal agent behavior on the species coexistence in competitive communities with and without habitat destruction could be explored. The model consists of four module components: dispersal rules, species competition, species colonization, and habitat destruction. An experimental simulation was conducted using three hypothetical species with differing competitive and migration abilities in both intact and disturbed conditions for 250 years. The findings of this study support the theoretical expectation that inferior competitors can coexist with superior competitors given that the inferior competitors have efficient colonization ability. The simulation also reveals the important role of agent behavior in the seed dispersal process and the biased impact of environment fragmentation on superior competitors that are not superior dispersers.
\end{abstract}

(C) 2007 Elsevier B.V. All rights reserved.

Keywords: Forest landscape modeling; GIS; CA model; Seed dispersal simulation; Dispersal agent behavior; Species competition; Habitat destruction

\section{Introduction}

The significance of spatial patterns, structures, and processes in landscape dynamics is well recognized. Landscape ecologists are applying ecological concepts to the study of land management systems and are discovering how and why space affects the environment (Malanson, 1993). Computational models are used to simulate spatial interactions in environmental processes that create the distribution of climates, life, and landforms on the earth. A computer simulation approach is necessary when experiments with a real landscape are constrained by temporal, regulatory, or other logistical reasons. Simulation models provide norms for comparison with real behavior, and the differences between model and reality provide the basis for improvement of our understanding of the general principles programmed into the models. An improved model can then be used to develop and evaluate different landscape transformation scenarios, as well as to answer "what-if" questions (Clarke and Gaydos, 1998).

\footnotetext{
* Corresponding author.

E-mail address: ffqiu@utdallas.edu (F. Qiu).
}

Many simulation models have been constructed to address the dynamics of meta-populations responding to habitat destruction in competitive communities by considering seed dispersal in space (Tilman et al., 1997; Dytham, 1994, 1995; Nee and May, 1992). According to Hanski (1994), the utilization of the spatial concept in these simulation models can be divided into different categories. Some of these models (Shugart and West, 1977; Nee and May, 1992; Ellison and Bedford, 1995; Tilman et al., 1994, 1997) do consider the spatial position of various species by assigning them to cells at different locations. Unfortunately, the interactions among cells are often treated equally under the assumption of ubiquitous dispersal; any species can arrive on any cell in the grid at any time, given suitable environmental conditions. These models are spatially implicit in that they retain the spatial aspects of the environmental conditions through environmental gradients, but they effectively ignore the spatial distribution of the population under consideration. Spatially explicit models (Dytham, 1995; Malanson and Cairns, 1997; Malanson and Armstrong, 1996; He and Mladenoff, 1999) either restrict the migration of species to the adjacent cells or allow distance-dependent colonization probabilities to be assigned to all the cells, so that the location of the lattice related to species source is explicitly taken into 
account. Other models (Hanski, 1994; Moilanen and Hanski, 1995) incorporate either population area or other characteristics of habitat patches, while distance remains an important factor. These models are considered to be better approximations of reality, but only the trees on the edge of the patch can have the opportunity for seed dispersal.

All of these models have incorporated spatial components in their simulations of species responses to habitat destruction during competitive coexistence; however, the results are qualitatively similar to, but quantitatively different from, each other. For example, two common inferences have often been drawn from these studies. First, inferior species, such as "weedy species," may coexist with a superior competitor capable of excluding its rival from any patch where it would colonize, given that the inferior competitor either disperses more effectively or has a lower extinction rate (Tilman et al., 1997; Dytham, 1995; Nee and May, 1992). Second, in the presence of patch removal, a "weedy species" that is an inferior competitor but better disperser may increase in abundance (Tilman et al., 1997; Dytham, 1994, 1995; Nee and May, 1992) as a result of habitat destruction. Consequently, a superior competitor may experience extinction first, earlier than an inferior competitor that has better colonizing capability and is more efficient in coping with habitat destruction.

Seed dispersal ability plays a key role in the establishment of species in a particular location in all the aforementioned models. As a decisive factor of the distribution of various species in space, seed dispersal determines the abundance of a species over a competitive environment. Spatially implicit models only consider the spatial structures of environment gradients and distances to the source based on the assumption of ubiquitous establishment without taking into account the different modes of dispersal processes (Waldron, 2002). Spatially explicit models, such as FORFLO (Hanson et al., 1990) and MOSEL (Malanson and Cairns, 1997; Malanson and Armstrong, 1996), explicitly take into consideration the spatial process in the simulation of species propagation by including several seed dispersal mechanisms, such as wind, gravity and animals, as input parameters. FORFLO (Hanson et al., 1990) models seed dispersal in a riparian environment. However, except for the fact the model differentiates flooded downstream plots from other plots, the seed dispersal probability for all agents was simply determined by a discrete distribution function that is essentially distance dependent. MOSEL improves upon the JABOWA-FORET model by applying a continuous distance decay function to calculate a dispersal probability of the species driven by each of the seed dispersal mechanisms. Another popular descendant of JABOWAFORET, the ZELIG model (Smith and Urban, 1988; Urban, 1990) allows plants to produce seeds inside the modeled plots and permits dispersal across grid cells. The SORTIE model (Pacala et al., 1993, 1996) incorporates more spatial interaction than other JABOWA-FORET-derived forestry models. SORTIE tracks individual tree locations and simulates seed dispersal by using mean dispersal distances and seedling densities for each species. In SORTIE, competition is modeled as a function of light only based on field observations suggesting competition for nutrient or moisture was not important compared with light. Instead of focus on the dispersal mechanism, SORTIE is more a forest growth model emphasizing on the calculation of available light to adapt for differing forest species, with a detail that makes it very computationally expensive (He and Mladenoff, 1999). As with aforementioned models, the seed dispersal procedure in these extended JABOWA-FORET models is also mostly distance-based, without built-in capability to characterize the behavioral differences and uniqueness of various dispersal agents, which are the ultimate driving force for the vegetation distribution in a landscape.

The most widely cited spatially explicit model in the literature is likely the LANDIS model (Mladenoff et al., 1996; Mladenoff and He, 1999; He et al., 1999; Gustafson et al., 2000). The LANDIS landscape model is capable of simulating forest landscape change over large heterogeneous spatial and temporal scales, by investigating the dynamics of seed dispersal, disturbance by wind, fire, and biological (insects and diseases) agents, harvesting, and fuel management. Within LANDIS, seedling establishment is determined by a seed's dispersal probability, which is a function of an exponential distribution, parameterized by the effective and maximum dispersal distances (He and Mladenoff, 1999). After dispersing, LANDIS uses light condition checking and site condition checking to see whether or not a seed is able to sprout successfully (He et al., 2005). LANDIS provides the ability to model various interactions within a forest landscape, with a complexity and robustness that could not be achieved by other models. Similar to other spatially explicit models, albeit with great improvement, the seed dispersal in LANDIS is still simulated using a distance dependent stochastic process, often resulting in isotropic distribution with radii of varied size for different species. Due to its emphasis on the simulation of relatively flat landscapes of large temporal and spatial scales, LANDIS does not necessarily pay close attention to anisotropic characteristics of a specific dispersal agent and the potential directional bias of the resultant vegetation distribution. The behavior characteristics of dispersal agents, however, are often the most critical elements in simulating the individual interactions among species at a local scale.

Any attempt to incorporate dispersal into a forest growth model should not ignore the spatial behavior of the agents upon which seed dispersal depends (Murray, 1986). van der Pijl (1982) identified the following major dispersal agents: zoochory (animal), anemochory (wind), hydrochory (water), and barochory (gravity). The spatial behavior of these dispersal agents plays a much more significant role than the distance to the seed source. Seed dispersal by anemochory (wind) is usually determined by the direction and speed of the prevailing wind rather than solely by the relative distance to the mature trees. In most cases, a location in an upwind direction will not receive any seeds from a tree and not every cell in the downwind direction is able to receive seeds. It is usually the strength of the wind that determines the maximum seed dispersal distance. Seed dispersal driven by barochory (gravity) is unlikely to happen in a position that has a higher elevation than the source tree except for immediately neighboring 
locations. Therefore, the underlying terrain topography should be included to model seed dispersal driven by barochory agent because it plays a dominant role in determining vegetation distribution in a landscape, especially in mountainous areas with a large topographical relief. Seed dispersal driven by hydrochory (water) is more sophisticated than those driven by barochory, because it is not only governed by gravity, but also restricted to the availability and the flow speed of water channels. Seed propagation via zoochory (animals) is usually affected not only by the seed source but also by animal habitat location and its activity corridor. The assumption that seed dispersibility is ubiquitous or uniform in all direction in these situations is unrealistic. In order to simulate the forest dynamics in a realistic manner, it is necessary to incorporate the spatial behaviors of the above seed dispersal agents in the models.

Among various simulation models in landscape ecology, only a few attempted to incorporate the above four seed dispersal agents in the modeling process. The MUMODIMO model (Waldron, 2002) is among the earliest efforts that strived to explicitly model the four major dispersal mechanisms to predict seed dispersal distance and direction at the landscape scale. The most impressive component of the model is its ability to simulate anemochory (wind) driven seed dispersal using wind velocity and vector data. The distribution of seed dispersal was mimicked with an altered ellipse, although the model does not seem to treat the upwind and downwind directions differently. Compared to anemochory, the simulation of other dispersal agents are rather simplistic according to the author. For example, the dispersal by water agent (hydrochory) only concerns floodplain and the river itself without taking into account of the flow direction of the water channel. The simulation of barochory does not utilize any topographic characteristics that fundamentally control the gravity driven dispersal, limiting its application to only a flat plain. Seed dispersal driven by zoochory is modeled simply either as movement to immediately neighboring cells (for mammals) or as a distance-based negative algebraic decay function between source and sink (for birds).

The objective of this paper is to develop a prototype of GISbased Spatially Explicit Model Of Dispersal Agent behavioR (SEMODAR) to simulate the behavioral characteristics of the four fundamental agents. The impacts of the agent behavior on the vegetation distribution of different species in competitive communities with and without the habitat destruction can therefore be investigated. A unique feature of this prototype model is that it was based on the state-of-the-art component object model (COM) technology and the most widely used GIS software system, ArcGIS. It is an extension and upgrade of an earlier model that we developed inside ArcInfo (a forerunner of ArcGIS) using the Arc Macro Language (AML) (Qiu, 1998). The development of such a model offers an alternative perspective in studying the coexistence of multiple species and their response to habitat destruction in a competitive environment.

The next section of the paper presents the background information related to seed dispersal models and the use of GIS in such models. This is followed by the detailed description of the proposed model and the development of the prototype software. An experimental simulation is presented to verify a common inference of previous studies, followed by discussion and conclusion.

\section{Background}

Various seed dispersal models have been built, and the majority of them are constructed using the cellular automaton (CA) approach. As a discrete spatio-temporal dynamical system, a CA is suitable for examining the nature of between-cell interaction, species migration and competition, flux of energy, matter and information, and population dynamics at the community level (Ellison and Bedford, 1995). The CA model was first developed by Ulam in the 1940s and adopted by Von Neumann to investigate the logical nature of self-reproducible systems (White and Engelen, 1993; Li and Yeh, 2002). Mathematically, CA models can be viewed as the discrete analogs of partial differential equations (Wolfram, 1986). CA models are usually defined by an action space, an initial configuration, and a set of behavior rules (Clarke and Gaydos, 1998; Takeyama and Couclelis, 1997). A CA model often divides the action space into grids with either regular or irregular cells, which contains a finite set of states for that particular location. The initial configuration specifies the starting states for all the cells in the action space. These states are updated by a set of rules acted upon a current cell or other cells in a neighborhood of specific size and shape. These rules are often called transition rules, because they determine the next state of a cell based on the current state of the cell and/or its neighboring cells through modeling iteration (Czárán, 1998). In a CA model, complex global patterns can emerge after many iterations of simulation by properly defined transitional rules (Batty and Xie, 1994).

While most of the popular seed dispersal models are constructed from scratch using a common programming language such as FORTRAN, $\mathrm{C}$ or $\mathrm{C}++$, SEMODAR is developed on top of a widely used geographic information system (GIS) product. Seed dispersal is an inherently geographic phenomenon because it involves the spread of objects over space through time (Waldron, 2002). Geographic information systems, especially raster-based GIS, share many striking similarities with CA model in data representation, manipulation, visualization and analysis (Wagner, 1997). Similar to that in GIS, the action space of a CA is usually configured as a grid with regular tessellation, and one cell of the tessellation can only hold one state (attribute) at a time for a specific variable. States of the cells for different variables in CA are organized into layers as in GIS. Input data collection and capturing (such as scanning and digitizing) for a $\mathrm{CA}$ is often the first step for any further modeling effort. Many of the required input datasets to a CA model, such as initial model state and other environment conditions like soil, land use, precipitation, and temperature data are widely available in GIS format, and there is little to no conversion necessary for these data. In addition to its ability to prepare input data for a CA model, GIS also provides the built-in capability to present modeling results 
and simulation dynamics as map displays. Compared with many standalone CA models, GIS is bestowed with powerful map rendering capabilities and convenient user interfaces that allow for a customized display of the model states in the form of a choropleth map in both two-dimensional and three-dimensional space. With embedded scripting and programming capabilities, contemporary GIS also offers the options to produce movie animations that can track the temporal succession of the whole vegetation regeneration process (including seed production, seed dispersal, seedling establishment, tree growth and senescence) of different species and their spatial interaction (such as vegetation distribution, competition, and coexistence).

GIS are equipped with many powerful spatial operations that can be used directly in a CA model. Most raster-based GIS provide ready-to-use local, focal, zonal and global operations in the form of map algebra (Tomlin, 1990). They correspond to CA operations at the plot, neighborhood, patch and landscape levels, respectively. Local operations allow the calculation of a new value for each cell based on the values of the corresponding cells of multiple layers of environment conditions using mathematical operations (e.g. addition, subtraction, multiplication, division, etc.) and functions (e.g. sine, cosine, mean, minimum, maximum, etc.). Local operations can be used to code transition rules that update the state of each plot based on multiple environment conditions. Focal operations determine the cell values as a function of all the values of a specific neighborhood of that cell. A typical neighborhood is a standard $3 \times 3$ rectangular moving window, equivalent to the Moore neighborhood in CA. A neighborhood in GIS has the flexibility of being customized with user defined shape, size and orientation to provide alternatives to the regular neighborhood in traditional CA models. Zonal operations derive value for each cell based on all the cells within the same zone. A zone, equivalent to a patch, is essentially a group of cells with the same attribute in the landscape. Global operations act on extended communities and can potentially expand the CA action space to the whole landscape. For example, the global pseudo-randomization function can be used to generate a randomized surface that is needed in most stochastic CA.

Apart from these four categories of basic operations, rasterbased GIS are also furnished with rich collections of application-specific operations that specialize in data analysis of certain domain areas. Examples of application specific operations include, but not limited to, distance-based cost analysis, terrain surface analysis and visualization, and hydrological modeling functions. These operations provide many valuable tools that can be used to code complex transition rules to simulate the unique and sophisticated behavioral characteristics of the aforementioned dispersal agents. For example, the distance-based cost analysis operations can be used to derive animal activity corridors between their habitats and food sources. When combined with the randomization surface generated with global functions, the distance-based cost functions can be utilized to compute distance-based dispersal probability, which is important to the stochastic modeling of all agent types. Most CA models that we encountered in the literature all utilize a two-dimensional grid as the underlying structure. The use of terrain surface analysis and visualization operations, however, makes it possible to build a threedimensional CA. The ability to model terrain features by employing digital elevation models (DEM) from a GIS allows for the simulation of complex gravity dispersal schemes, which can extend the action space of current seed dispersal models from flat plains to areas with a great topographical relief. Numerous hydrological modeling operations that are now available in many GIS can derive water channel networks and stream flow direction using DEMs, making it possible to simulate complicated dispersal process of hydrochory agents.

Takeyama and Couclelis (1997) were the first to demonstrate the potential of GIS in CA modeling. In doing so, they proposed to bridge the gap between map-based spatial manipulation and equation-based spatial modeling. Other research has shown that GIS provides an inherent ability to relax the strict local neighborhoods that are necessary for natural science CA models (Batty et al., 1997; Takeyama and Couclelis, 1997; Wu, 1999). By using a more relaxed approach, the model is able to allow for more flexible spatial interactions. The use of irregular neighborhoods is regarded to be more powerful in terms of defining custom action space for a specific agent type.

\section{Model description}

SEMODAR consists of four components: the agent dispersal transition rule, species competition, species colonization and habitat destruction. The agent dispersal rule module delineates the transition rule of each species involved in their possible dispersal space. The species competition module determines the dominant species if more than one species is able to reach a site. The species colonization module uses a stochastic distance decay function to determine the actual establishment of a species in a plot. The habitat destruction module will eliminate a seed from a site if environmental degradation due to soil erosion, insect or disease infection occurs at that site.

Models seeking to improve our understanding of the behavior of a process through simulation are usually based on a relatively simplified representation of the reality under certain prescribed assumptions. To concentrate just on the investigation of the effects of agent behavior on the dispersal process and species response to environmental disturbances, we also made several assumptions. On the one hand, these assumptions are set so that the prototype model can be straightforward enough for us to conduct a "proof-of-concept" study. On the other hand, they are chosen so that they can be analogous to established literature (Nee and May, 1992; Moilanen and Hanski, 1995; Ellison and Bedford, 1995), except for the fact that the ubiquitous and uniformed assumptions do not apply anymore. These assumptions also provide the initial model configuration upon which customization of the model is possible through user parameterization.

Some of the environmental variables, such as soil, precipitation, and temperature are regarded as irrelevant in the growth processes and are not included in this prototype model. For a simulation of a local scale, there exists only small 
gradient of these environmental conditions. It is therefore assumed that any species can grow within a location if it manages to arrive at that location, and it will not perish unless it experiences habitat destruction, reaches a predefined maximum living age, or is pushed-out by a superior competitor. From this perspective, the SEMODAR model is not a full forest growth but more of a seed dispersal simulation model. Each species is associated with a generation time and a maximum living age. Generation time of a species is defined as the number of years from its seed germination to seed production. This means that a species cannot produce seeds immediately after its arrival at a particular cell. This lag time between its establishment and reproduction is critical to the overall rate of seed dispersal (Malanson and Cairns, 1997). The maximum living age specifies the time beyond which a tree will perish and lose its ability to reproduce seeds. Moreover, each cell can only host one species at any moment, though more than one tree of this species can persist in the unit. The dominance of a species in a cell is dependent upon the outcome of species competition and seed dispersibility.

\subsection{Dispersal transition rule}

In order to model the various dispersal processes driven by different agents, a detailed understanding of the agent behaviors that govern the seed distribution of each species is necessary. At present, two ubiquitous processes (wind and gravity) out of the four migration mechanisms were selected and implemented as major determinants of seed distribution, because their data are more widely available compared to the other two agents (i.e. animal and water). The seed dispersal of a species in the prototype model can be configured to be driven by either wind agent only or gravity agent only, or can be driven by both agents. Seed dispersal by wind is usually more efficient than that by gravity because of the mobile nature of the wind agent and the restraints placed upon the gravity agent by the topography.

\subsubsection{Wind agent}

Wind has been modeled as a disturbance to growth and a dispersal agent in forest landscape models. In disturbance models, such as LANDIS (Mladenoff and He, 1999), wind is primarily modeled as a direct disturbance, through high-speed shearing, or as an indirect disturbance, creating debris as potential fuel for a forest fire. Wind in this research, however, is employed as a dispersal agent. Seed dispersal by wind, the wind-driven movement of seeds along the ground and through the air, is an important mechanism for dispersal in plant species in various environments (Schurr et al., 2005). Wind is able to carry seeds to very distant locations from their sources, depending on the size of the seeds. The most obvious characteristics of wind are direction and strength. The direction of a wind is usually described by its angle from the north direction and its strength by the velocity (speed). Due to the fact that seed dispersion by wind is unevenly distributed in different directions and distances from the seed sources, it is more appropriate to represent wind driven seed dispersion as an

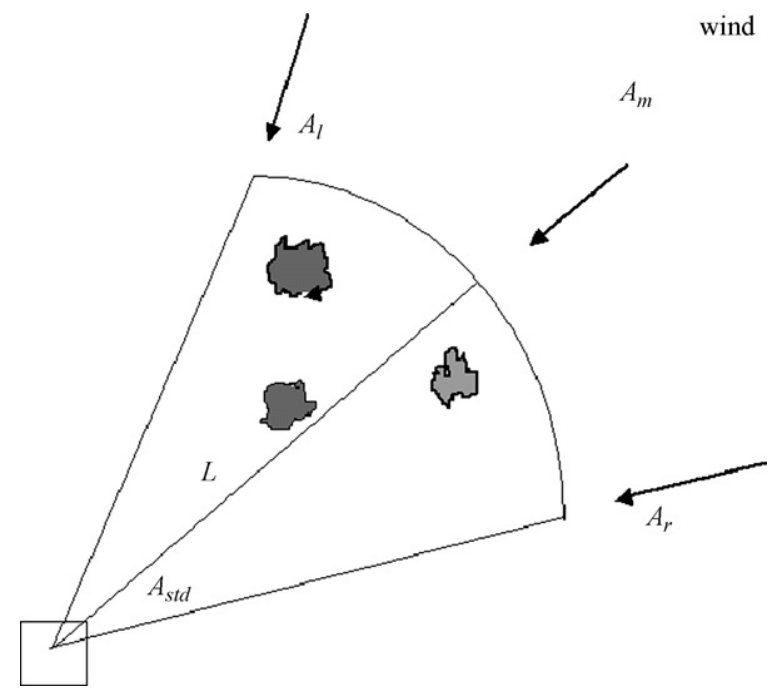

Fig. 1. Simulation of seed dispersal by wind using a wedge-shaped moving window with its wide end situated towards the wind, where $A_{1}$ and $A_{\mathrm{r}}$ are the two angles, and $A_{\text {std }}$ is the standard deviation and $A_{\mathrm{m}}$ is the mean direction angle and $L$ (length) of the wedge represents the maximum distance a seed can be possibly carried by wind.

asymmetrical spatial distribution instead of symmetric ones such as rectangle and circle. Unlike the MUMODIMO model (Waldron, 2002) that simulates the wind driven seed dispersal as an altered ellipse, SEMODAR uses a wedge-shaped neighborhood, which is much easier to define by simply specifying its length and the directional angles of its two edges. Used as an irregular neighborhood moving across the whole grid, SEMODAR treats the narrow end of the wedge as the neighborhood's focus (the current cell under consideration for the establishment of seeds) as in raster-based GIS. The wedgeshaped neighborhood is oriented with its wide end situated towards the wind so that only mature trees upwind of the current focus falling within the wedge neighborhood have the potential to disperse seeds into the focus cell (Fig. 1). For neighborhoods with mature trees of more than one species, only the seeds of the most competitive species can survive in the focus cell.

The length and the two angles of the wedge are calculated based on the observed weather station data at each location over a period of time. The angular mean and the standard deviation statistics of the prevailing wind directions needed to be first calculated. Since angles are measured on a circular scale instead of a linear scale, the angular mean and standard deviation were computed using the vector statistical method developed by Hodgson and Gaile (1996). Instead of using simple arithmetic statistics, the vector statistical method computes the vector mean, the vector standard deviation and the mean length of the wind vectors. Once the standard deviation and the mean of the prevailing wind direction are determined, the two angles of the wedge can then be expressed as in Eqs. (3.1) and (3.2), where $A_{1}$ and $A_{\mathrm{r}}$ are the two angles, and $A_{\text {std }}$ is the standard deviation and $A_{\mathrm{m}}$ is the mean direction angle. The length $(L)$ of the wedge represents the maximum distance a seed can be possibly carried by wind. It is a function of the mean wind velocity $\left(V_{\mathrm{m}}\right)$ and the seed size dependent 
dispersal scale rate by wind $\left(R_{\mathrm{w}}\right)$ as expressed in Eq. (3.3).

$A_{1}=A_{\mathrm{m}}-A_{\text {std }}$

$A_{\mathrm{r}}=A_{\mathrm{m}}+A_{\mathrm{std}}$

$L=V_{\mathrm{m}} \times R_{\mathrm{w}}$

This method ensures that the cells in the prevailing upwind direction have the highest chance to disperse seeds to the focus cell. Only in an extreme case, when the wind direction at a location is very diverse, the standard deviation will be $180^{\circ}$ and the wedge will become a circle so that trees in any direction have a similar chance of sending seeds to the focus cell. Unlike many other spatially explicit models that allow any seed to arrive at any location with a certain probability, this method prohibits seed movements across barriers wider than wedge length (i.e. the maximum distance that the seed can travel) such as the case of a big lake. Thus, the impact of landscape fragmentation on seed dispersal can be modeled.

\subsubsection{Gravity agent}

The gravity agent causes seeds to drop to lower elevations in a landscape. A fundamental behavioral characteristic of the gravity agent is that all objects driven by gravity cannot move from locations of lower elevation to locations of higher elevation. Therefore, locations with altitudes higher than the seed source will not be able to receive any seed dispersed from that source by gravity only. Since topography and the elevation of the cells relative to that of the source trees dominate the seed dispersal process by gravity, it is imperative that a digital elevation model (DEM) be used in the model to determine the locations that can possibly receive seeds from trees at higher positions.

Seeds dispersed by gravity always reach the immediate vicinity of their source trees first. The seeds from all directions have the same opportunity to arrive at a cell if these seeds have the same higher altitude than that cell. Thus, a symmetric $3 \times 3$ rectangular moving window was chosen to simulate the behaviors of gravity agent in the model. The focus of the moving window is the center of the rectangular neighborhood in GIS, and is the cell currently under consideration for the establishment of seeds. The locations of the other eight neighboring cells in the rectangular window are referenced by their relative cell coordinates to the current focus in the GIS (Fig. 2a). For example, the cell to the east of the current cell is represented by the neighborhood notation $(1,0)$ and the cell northwest of the focus is referenced by the notation $(-1,-1)$. In order to determine whether a neighboring cell has a potential for dispersing seeds into the current focus, we have to calculate the difference in elevation between the neighboring cell and the current focus. For example, the difference of elevation $\left(\operatorname{Elv}_{\mathrm{d}}\right)$ between the northwest cell $\operatorname{Elv}(-1,-1)$ and current focus $\operatorname{Elv}(0,0)$ is calculated as:

$\operatorname{Elv}_{\mathrm{d}}=\operatorname{Elv}(-1,-1)-\operatorname{Elv}(0,0)$

Suppose this difference in elevation between the northwest cell and the focus is greater than a predefined minimum value for
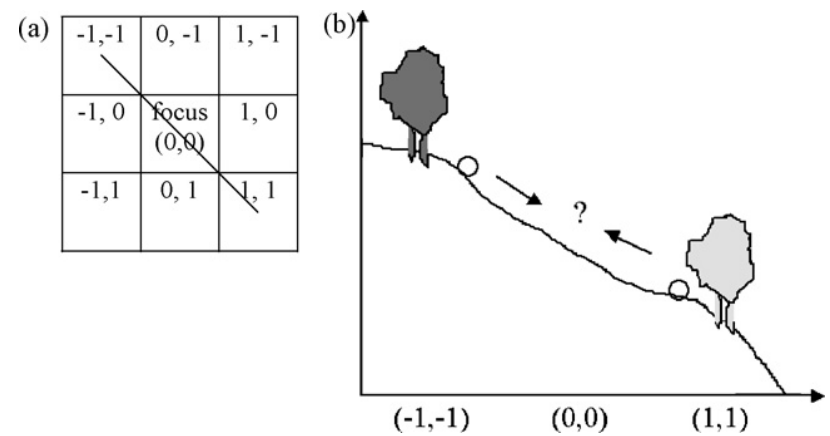

Fig. 2. Simulation of seed dispersal by gravity using a $3 \times 3$ moving window. (a) Neighborhood notation of a $3 \times 3$ moving window used to reference the neighboring cells. (b) Elevation profile from cell $(-1,-1)$ to cell $(1,1)$, where "?" stands for the location of the focus, which may or may not receive seeds from immediate neighboring cells depending its relative elevation.

the species and there is also a mature tree of a species that is capable of producing see in the northwest cell, it is possible that the focus cell can receive seeds dispersed from that cell. In another example, if the elevation difference between the southeast cell and the focus cell is less than the predefined minimum value, it is impossible for the neighboring cell to disperse seed into the focus cell. Fig. $2 b$ gives a profile diagram along cell $(-1,-1)$, focus cell $(0,0)$ to cell $(1,1)$ illustrating the above situations. In the case that more than one neighboring cell is higher in elevation than the center cell, which cell will contribute to the final establishment of a seed in the focus will be determined by the outcome of species competition and colonization, which will be discussed later.

The $3 \times 3$ moving window limits gravity-driven seed dispersal to only the immediate neighbors of the focus cell with a dispersal distance of only one cell size. In reality, a seed can roll down more than one cell to a distant location of a lower elevation. Increasing the window size from 3 to 5 cells or more seems to be a solution. There is a high risk, however, of violating the fundamental behavioral principle of the gravity agent. Taking a $5 \times 5$ window as an example (Fig. $3 a$ ), one may observe that the elevation in cell $(-2,-2)$ is higher than that of (a)

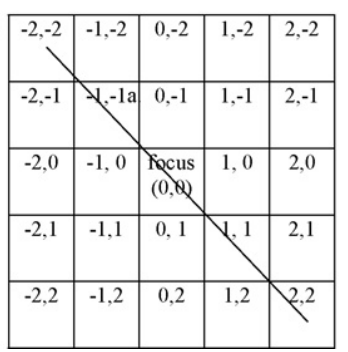

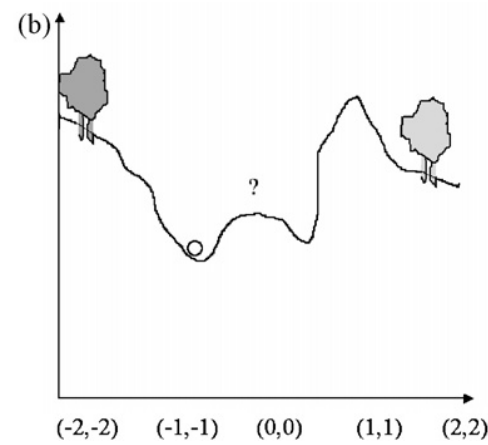

Fig. 3. Simulation of seed dispersal by gravity using a $5 \times 5$ moving window having a high risk of violating gravity dispersal rules. (a) Neighborhood notation in a $5 \times 5$ moving window used to reference neighboring cells. (b) Elevation profile from cell $(-2,-2)$ to cell $(2,2)$, where "?" stands for the focus of the $5 \times 5$ moving window, which may not receive a seed from an extended neighbor because seeds can be easily trapped in local valleys, or blocked by local hills defined by immediate neighbors. 
the focus $(0,0)$. As a result, one might rush into an inappropriate conclusion that it is possible for cell $(-2,-2)$ to disperse seed to the focus by gravity. A careful examination of the topographic characteristics of the neighboring cells along the profile (Fig. 3b) would show that there exists no such possibility because the elevation at cell $(-1,-1)$ is lower than that at the focus. The seeds will stop at this cell and will not reach the focus if only the gravity agent is considered in the process. A similar situation happens when, for example, cell $(2,2)$ is higher than the focus cell but lower than cell $(1,1)$ in the window. The seed in cell $(2,2)$ will not be able to climb the hill defined by cell $(1,1)$ to get to the focus cell if dispersal is only driven by gravity (Fig. 3b). Fig. 3b demonstrates that seeds dispersed by gravity may easily get trapped in local valleys and enlarging the size of neighborhood does appear to be a good solution.

We propose an alternative approach to breaking the one cell dispersal distance limit by running the $3 \times 3$ window several passes in each seed dispersal iteration. For example, a two-pass simulation with a $3 \times 3$ window will resemble that with a $5 \times 5$ moving window (Fig. 3a). The attraction of this alternative method is that the fundamental behavioral rule of the gravity agent is perfectly preserved and the possible risks inherent in simulation using a $5 \times 5$ or larger window can be avoided. Within one seed dispersal season, a species can experience many passes of a $3 \times 3$ moving window. The total number of moves that a species can have will determine the maximum possible dispersal distance of a seed driven by the gravity agent.

\subsection{Species competition}

Species competition determines which species will potentially be established in a cell when more than one competing species can disperse seed in the cell. Based on the assumption that a cell can host only one species at a time, it is stipulated that a superior competitor with a higher rank can invade a cell occupied by an inferior competitor and exclude the inferior competitor from that cell. An inferior competitor, on the other hand, cannot encroach upon a cell already established by a superior competitor even if the inferior competitor has higher colonization efficiency. In this research, the competitive ability of the species is defined by ranking all species under study hierarchically from the poorest competitor to the most successful competitor in an ascending order. If there are species to be simulated in the model, each species will be given a unique superiority rank of competition between 1 and $n$ with 1 standing for the poorest competitor (species 1 ) and $n$ standing for the best competitor ( $\operatorname{species} n$ ). Species $n$ is able to exclude all other species from any site that it invades. Species $n-1$, the second best competitor, can displace all other species except the best species. Species 1, the poorest competitor, can be displaced by any other invading species. To determine which species will be established in the focus of a neighborhood, the species with the highest rank in the neighboring cells is chosen as a candidate species. If the focus is an empty and habitable cell, then this candidate species has the possibility to disperse seed into the cell. If the focus is not empty, the candidate species will compare its competitive rank with the species that has already been established in the focus. The candidate species may displace the original species and occupy the focus if it is a better competitor. Otherwise, the original species in the cell will stay without being replaced. If the focus cell is uninhabitable because of environment disturbance or degradation, then no seed of any species can be established there.

\subsection{Species colonization}

The dispersal agent rule and competitive hierarchy can help to decide whether a seed of a neighboring cell has the possibility to arrive at the focus cell and which seed of different species can potentially occupy of the focus cell if there is more than one possibility. However, the real establishment of a species in the focus cell is determined by the species' colonizing ability. The colonization of a species in a landscape is affected by its generation age, maximum living age and most importantly, its seed dispersal ability. A species with a shorter generation time and a longer maximum living age, and better dispersal ability is more efficient in the colonization process.

Unlike spatially implicit models which consider only the distance to the source tree, but similar to some of the spatially explicit models (such as LANDIS and MUMODIMO models), SEMODAR simulates the seed dispersal of a species as a stochastic process. The seed dispersal ability is simply codetermined by the seed's maximum dispersal probability $\left(P_{\mathrm{m}}\right)$ and maximum dispersal distance $\left(D_{\mathrm{m}}\right)$. The maximum dispersal probability of a species is the probability for the seed to be established in the cells immediately adjacent to the source tree. Unlike LANDIS, the maximum dispersal probability is not a fixed number such as 0.95 and can be customized according to type of the species. Additionally, the current version of SEMODAR does support the concept of effective seeding distance as used in LANDIS. The maximum dispersal distance is the distance beyond which all the cells will have zero seed establishment probability. Therefore, the cells in between the maximum dispersal distance and the seed source may have a seed establishment probability between 0 and $P_{\mathrm{m}}$. The calculation of maximum dispersal distance is related to the seed dispersal agents involved. If a species is driven only by wind, then $D_{\mathrm{m}}$ is equal to the length of the wind wedge as calculated in Eq. (3.3). If the only dispersal agent of a species is gravity, then the $D_{\mathrm{m}}$ of this species will be assigned as the maximum moving distance determined by the total number of passes of the $3 \times 3$ window associated with the species. The more passes taken by the moving window leads to a greater maximum moving distance. In the case that the seed of a species can be dispersed by both wind and gravity, $D_{\mathrm{m}}$ is the summation of the wind wedge length and the maximum moving distance by gravity.

The probability $(P)$ of a species being established in a cell is then computed simply as a linear function of the cell's distance from its source $(D)$, the maximum dispersal probability $\left(P_{\mathrm{m}}\right)$ and the maximum dispersal distance $\left(D_{\mathrm{m}}\right)$ of the species:

$P=\frac{D_{\mathrm{m}}-D}{D_{\mathrm{m}}} \times P_{\mathrm{m}}$ 
where $D$ will be forced to equal $D_{\mathrm{m}}$ if it is greater than $D_{\mathrm{m}} . P$ is therefore a normalized distance decay function in the range between 0 and $P_{\mathrm{m}}$. When the cell is at the maximum dispersal distance or beyond $\left(D \geq D_{\mathrm{m}}\right), P$ becomes 0 ; while the cell is at the closest distance $(D=0), P$ becomes $P_{\mathrm{m}}$, the maximum dispersal probability. $P$ is then used as an important criterion in determining the actual establishment of a species in a cell. Since the settlement of the seed at that location is a stochastic process in reality, a high seed dispersal probability does not guarantee the establishment of the seed in that cell. The actual establishment a seed in each cell is determined randomly based on the dispersal probability $(P)$ derived. Instead of using a universal threshold, a pseudo-random surface with values normally distributed between 0 and 1 was created using a random raster grid generator function. The random number generated for each cell of this surface is then compared with the seed dispersal probability $(P)$ surface for that cell. If the random value generated in a cell is less than the $P$ value of the cell, then the species will be established there. Otherwise, the species will lose its potential to grow at that location. Consequently, the higher the $P$ value of a species in a cell, the more likely it is greater than the random number generated and the greater the probability a seed of the species can establish there. The normally distributed nature of the pseudo-random surface ensures that, for example, for all the cells that have a dispersal probability of 0.5 , only approximately less than half of them will actually receive seeds from the species and allow the seeds to grow there.

\subsection{Habitat destruction}

To study the response of species to habitat destruction caused by environment disturbance, such as fire and insects, the model allows habitat destruction to be assigned to the cells of the CA model. Note that we do not model a specific disturbance force in the model yet, and we simply assume the habitat destruction occurs randomly in the landscape. To compare the differences in outcomes that may be caused by habitat destruction, the simulation can be conducted under an intact environment with all the cells available for the establishment of seeds or under a disturbed environment where cells are progressively excluded from the landscape as destroyed habitats. In an intact environment, the distribution of all the species will be primarily determined by the inter-species interaction. In this case, all species are competing for a single limited resource, i.e. the growing space. Theoretically, if the simulation is allowed to continue with adequate time, the best competitor will eventually displace all the other species and take over the entire community. The trade-off between competitive ability and seed dispersibility, however, will allow a stable coexistence between inferior and superior competitors for a long period of time (Tilman et al., 1997).

The simulation can also be performed under a disturbed environment, where at each subsequent year, a certain amount of random habitat destruction will occur. For example, the model can be configured, for example, so that approximately $10 \%$ of the cells in the whole landscape will randomly experience permanent destruction per iteration. Since newly selected habitat destruction will also likely occur in the cells that were previously destroyed, the actual destruction rate on the remnant community is much less than $10 \%$ per year. Therefore, the whole landscape would not be destroyed in just 10 years. As a matter of fact, the average destruction rate is usually less than $1 \%$ per year, as it often takes more than 100-200 years to demolish the whole landscape. In the presence of habitat destruction, the dynamic of the species population will no longer solely be decided by the species dispersal ability and inter-species competition for living space. Habitat destruction and the accompanying fragmentation of the environment are expected to exert a significant influence on the seed dispersal process. The introduction of barriers in the area as a result would impose different effects on various dispersal agents and lead to a biased extinction of the species involved (Tilman et al., 1997).

\section{Model development}

The SEMODAR model was written with the ArcGIS software development kit (SDK) using Visual Basic, one of the most popular program languages that support the component object model (COM) technology. ArcGIS SDK is composed of hundreds and thousands of ArcObjects, which are COM objects that expose all the functionality used to build ArcGIS. He et al. (2002) discussed the benefits of component-based modeling in ecological research. They emphasize that a component-based approach provides the ability to break down a potentially monolithic program into smaller, more functional components. They also underscore the extensibility of component-based model. In this research, the use of component-based model is taken a step further. Besides the capability to create module components and application extensions that can be plugged into ArcGIS or other standalone applications, COM allows full access to all the powerful spatial analysis abilities in GIS through ArcObjects, so that the spatial characteristics of the agent behavior can be modeled. Additionally, the use of components permits the collaboration of developers within different development environments (Java, .Net, C++, and other COM compatible language).

A standalone prototype program was developed based on the module components that were designed to realize the four modules described above. Fig. 4 displays the typical user interface of the program. This specific program window contains a modeling configuration window, two $2 \mathrm{D}$ visualization windows displaying the animation of the simulation progress (one with and the other without habitat destruction), and one $3 \mathrm{D}$ visualization window allowing the navigation of gravity driven seed dispersal in three-dimension space. The main program window contains a menu bar and a tool bar. The tool bar has standard Windows tools, while the menu bar consists of the following menus: File, View, Operations, Run, Visualization, Window and Help (Fig. 5). The File menu contains commands that allow users to load a previously saved project file (Open Project), to store model configuration parameters, paths to final and intermediate results files, etc. to a new project file (Save Project), to rename an existing project 


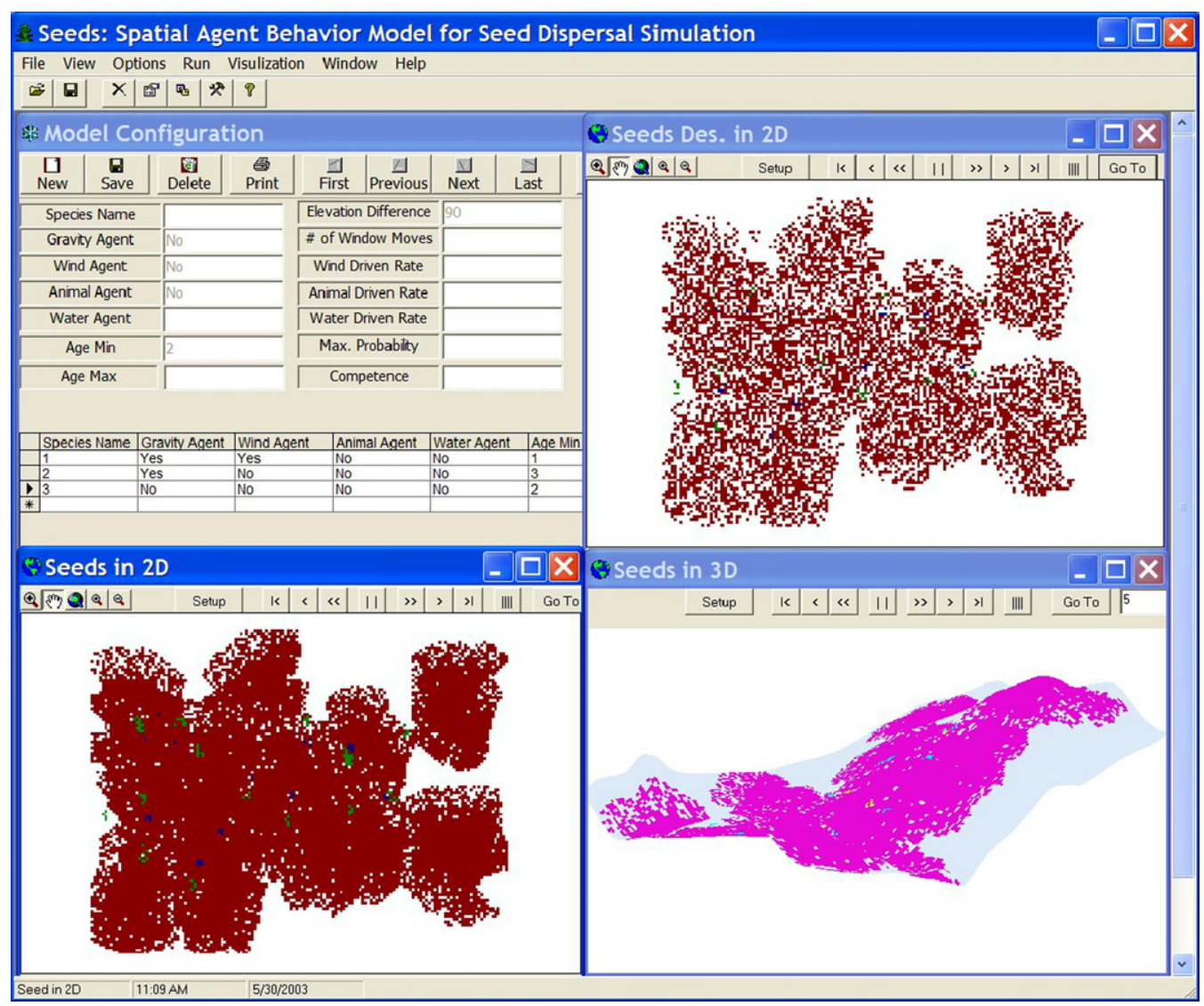

Fig. 4. The typical user interface for a standalone prototype program of SEMODAR.

file (Save Project As), to close all sub-windows inside the program and shutdown the main program (Close All Windows). The Export Grid command provides an option that enables the user to export any simulation progress grid shown on the visualization window to a JPEG image for external display. The View menu has two options that can show or hide Tool Bar and Status Bar. The Option menu is the place where the user specifies model parameters. The Initialization command defines the initial spatial distribution of seed sources (i.e. the initial state of the model). The seed sources can be generated randomly across a landscape or be obtained from a GIS grid file specifying the real distribution of existing tree species on a landscape. If the model involved the simulation of gravity driven dispersals, it is necessary to use the Load DEM command to define the topography of the landscape by a DEM file. The Model Configuration command launches the model configuration model window (as seen in Fig. 4) to define all important parameters of the model, such as species name, types of dispersal agents (animal and water agent types are still under development), generation age, maximum age. For elevation

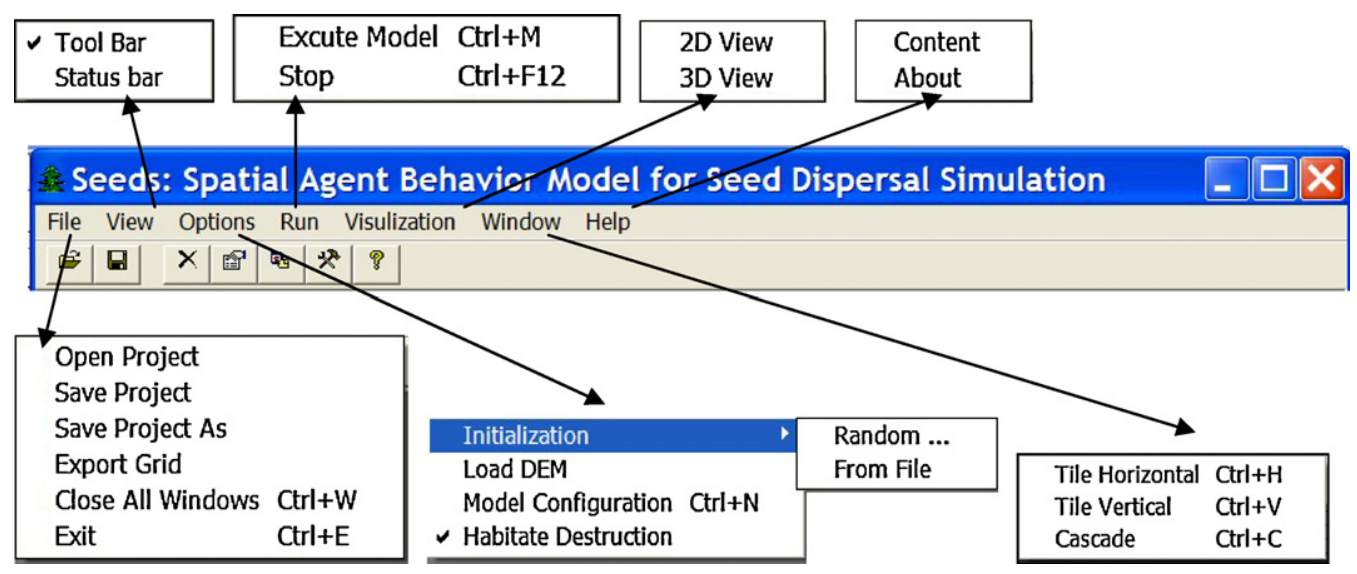

Fig. 5. The menus and commands for the standalone prototype program. 
driven dispersal, the minimum elevation difference needs to be defined and the colonization ability for the species are set by using the number of window moves per iteration. For other types of dispersal, the colonization ability for each agent type is specified by giving a scale rate. Finally, the competitive rank in the competence hierarchy for the species needs to be specified. The Run menu has two functions: Execute Model and Stop. Execute Model runs the model, but before it does, it prompts the user for the number of years to run. Stop can then cease the model from running at any time. The Visualization menu contains two options, $2 D$ View and $3 D$ View, which are viewers that can display the simulation progress and final result in either 2D or 3D mode (Fig. 5). Both views have their own tool bars. The 2D view has standard map navigation tools, such as Zoom In, Zoom Out, Select Area, Pan, and View Full Extent. The 3D navigation is controlled by mouse movement as in ArcGIS. Both views also have a Setup button that will bring up a window to define visualization animation parameters such as the start and end years, and the time interval. The $\mid<$ button displays simulation progress of the first year specified in Setup window. The $<$ button rewinds the progress at one time interval per click. The $\ll$ button displays the progress continuously backward. The $\|$ button pauses the animation temporarily. The three buttons $(\gg,>,>\mid)$ after the pause button are similar to the three before it, but are for animation forward instead backward. The ||| button stops the animation activity and resets it to the first year. The Go To button allows the user to jump to a specific year. The Window menu has three options, Tile Horizontal, Tile Vertical and Cascade, which are typical Windows operations to rearrange the windows opened in the program. The Help menu provides a table of Content that offers online help documents, and the About command provides the program version and copyright information.

\section{Experimental simulation and discussion}

To test the prototype model in its ability to simulate dispersal agent behavior, a pilot study was conducted to verify a common theoretical statement that seed dispersal ability is crucial for an inferior competitor to survive in a competitive landscape. Many articles in established literature (Tilman et al., 1997; Dytham, 1994, 1995; Nee and May, 1992) suggest that a competitively inferior species will be able to coexist with superior competitors for a long time in an intact community if it is a better disperser. Environmental degradation may favor an inferior competitor given that it has a fugitive migration style. Habitat destruction may drive the superior competitor to extinction first if it is a poor colonizer. We anticipated that the results from the pilot study would be analogous to those of the above literature, although different simulation mechanisms are employed.

A study area in Silver, Colorado was selected to conduct the pilot study. This study area is a mountainous region consists of a grid of $118 \times 150$ cells with a cell size of $30 \mathrm{~m} \times 30 \mathrm{~m}$, resulting in a total simulated area of $15.93 \mathrm{~km}^{2}$. A digital elevation model (DEM) of the study area was used as an input to simulate gravitydriven seed dispersal. The DEM shows an altitude range from 2749 to $3981 \mathrm{~m}$, indicating a large topographic relief.
Table 1

The model parameters used to simulate seed dispersal in the pilot study

\begin{tabular}{llll}
\hline Parameters of the model & Species 1 & Species 2 & Species3 \\
\hline Competence & 1 & 2 & 3 \\
Generation time/age min. (Year) & 1 & 3 & 2 \\
Maximum age/age max. (Year) & 100 & 80 & 90 \\
Maximum dispersal probability & 0.8 & 0.3 & 0.5 \\
Wind agent & Yes & No & Yes \\
Wind driven rate & 2.0 & N/A & 0.5 \\
Gravity agent & Yes & Yes & No \\
Minimum elevation difference (m) & 0 & 10 & N/A \\
\# of window moves & 4 & 2 & N/A \\
\hline
\end{tabular}

In the pilot study, we only chose three hypothetical species with different competitive and migration abilities, although the model itself can simulate a potentially large number of competing species. To study the behavioral characteristics of various dispersal agents and their response to habitat destruction, each species is assigned different competitive and colonizing abilities, as depicted by the model parameters listed in Table 1. Species 3 is defined as the best competitor, species 2 the second best competitor, and species 1 , the poorest competitor. With regard to the seed dispersal agent, species 3 is driven only by wind, species 2 is driven only by gravity, and species 1 is driven by both wind and gravity. Species 1 is the best disperser, species 3 is the second best disperser, and species 2 is the poorest disperser, based on their dispersal agent types, as well as other parameters, including generation time, maximum living age, and maximum dispersal probability. For species with wind-driven agent, the wind-driven rate is used to define its colonizing ability, and for species driven by gravity, the minimum elevation difference and the number of $3 \times 3$ window moves are used. Wind parameters such as wedge angles and length are calculated by using circular statistics based on weather station information over a 10-year period. Since it is difficult to get the wind information for all the years, we had to use the 10-year statistics repeatedly in the simulation.

The seed dispersal processes of these different species were simulated in a yearly-based time series. The model's output from the previous year is used as the input for the next year's simulation. The model was run for a maximum of 250 years using the same parameters both under an undisturbed environment and a disturbed environment with habitat destruction. The model keeps track of the age of the trees in each year. When the age of a tree becomes older than its generation time, it is labeled as being eligible to produce seed. When a tree reaches its maximum living age, it is removed from the cell. The cell, once occupied, becomes an empty habitable site for new seed germination.

The simulation without any habitat destruction was run first for 250 years and the extinction years of the three hypothetic species are listed in Table 2. Fig. 6 shows some of the snapshots of the simulation progress. The initial configuration (Fig. 6a) gives the seed sources distribution of the three species, with species 1 in black color, species 2 in dark gray and species 3 in light gray. At year 5 (Fig. 6b), species 1, the poorest competitor but the best disperser, driven by both wind and gravity agents, 
Table 2

The length of time until extinction of the three hypothetical species in years

\begin{tabular}{lcc}
\hline & Undisturbed environment & Habitat destruction \\
\hline Species 1 & 223 & 115 \\
Species 2 & 57 & 41 \\
Species 3 & 250 & 70 \\
\hline
\end{tabular}

colonized the majority of the landscape, while both species 2 and 3 had a very limited occupancy. Fig. 6c shows the simulation at year 25 , which reveals that species 1 was able to take all the remnant empty habitats in the landscape, but much of its previously established cells in the center of the landscape were invaded by species 3, the best competitor and the second best disperser. Species 2, the second best competitor and the poorest disperser, was also able to encroach into some of the cells occupied earlier by species 1 . However, species 2 only managed to colonize a small number of habitats, because it is constantly excluded by species 3 , and at the same time, is limited by its gravity only dispersal agent that constrains its migration to locations of lower elevation (Fig. 6c). At year 57 (Fig. 6d), species 2, although a better competitor than species 1, become the first to perish. Species 1, the poorest competitor but the best colonizer, coexisted with species 3 for a long time, but went extinct in year 223 when species 3 took over the entire community. As a result, the extinction of species 1 was much later than species 2 . Since only species 3 remained in the landscape at year 223, and all other species were excluded from the landscape as an outcome of a long-term competition, we did not include a map for year 223 in Fig. 6.

The interpretation of these results is that inter-species competition is the predominant factor in a closed and undisturbed community. Eventually, given enough time, the best competitor will eliminate all the other species, regardless of their seed dispersibilities. However, it is the species colonizing capability that determines the sequence of the extinction of the inferior competitors. As a consequence, the order of extinction for competitively inferior species varies according to their maximum dispersal distances and dispersal mechanisms. Species 2, which is only gravity dispersed, is easily constrained by the topography to the vicinity surrounding the seed source and hence is prone to extinction first if it is adjacent to superior competitors. Species 1, with the additional dispersal agent of wind, can escape eradication by a better competitor and can stably coexist with it for a long period of time because of the relatively mobile nature of its dispersal mechanism.

The model was also executed under conditions of progressive habitat destruction (Fig. 7 and Table 2). The same initial configuration for undisturbed environment (Fig. 6a) was used to specify the initial spatial distribution of the seed sources of the three species, so the simulation with and without habitat destruction can be compared. The same color legend with species 1 in black color, species 2 in dark gray and species 3 light gray is adopted in Fig. 7. At age 5 (Fig. 7a), a similar overall spatial pattern to that without habitat destruction (Fig. 6b) is observed, with species 1, the best disperser and the poorest competitor, having colonized the majority of the landscape. What was different was that the landscape started to become fragmented due to the effects of habitat destruction. Fig. $7 b$ shows the model simulation at year 20, where the three species still coexisted in a more fragmented landscape. Species 1 was still the dominant vegetation distribution, but much of its previously occupied habitats were lost to habitat destruction and species 3 . Species 2, the poorest disperser, only became established in a few plots at year 20, making it hard to be visible

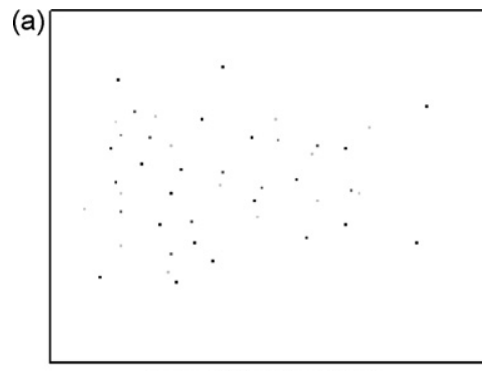

Initial Configuration

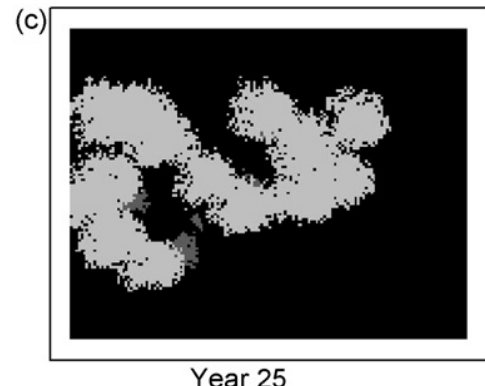

Year 25

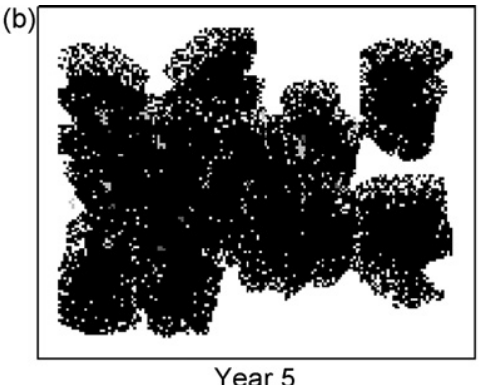

Year 5

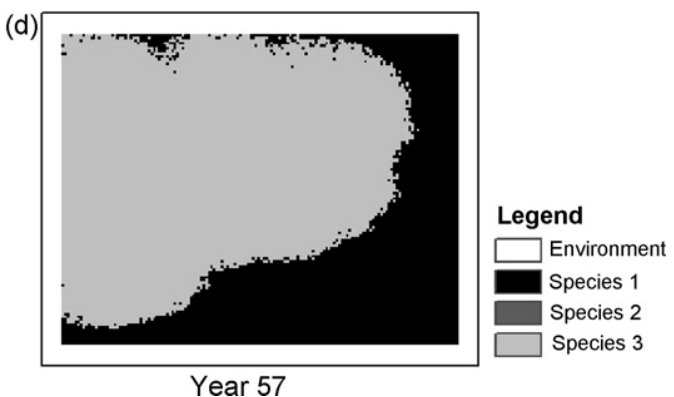

Fig. 6. The results of a 250 year seed dispersal simulation without environmental disturbance. (a) The initial configuration showing the seed sources distribution of the three species. (b) At year 5, species 1, colonized the majority of the landscape, while both species 2 and 3 had a very limited occupancy. (c) At year 25 , species 1 was able to take all the remnant empty habitats, with much of its previously established cells invaded by species 3 , and some by species 2 . (d) At year 57 , species 2 became the first to perish. Species 1 coexisted with species 3 until year 223 . 

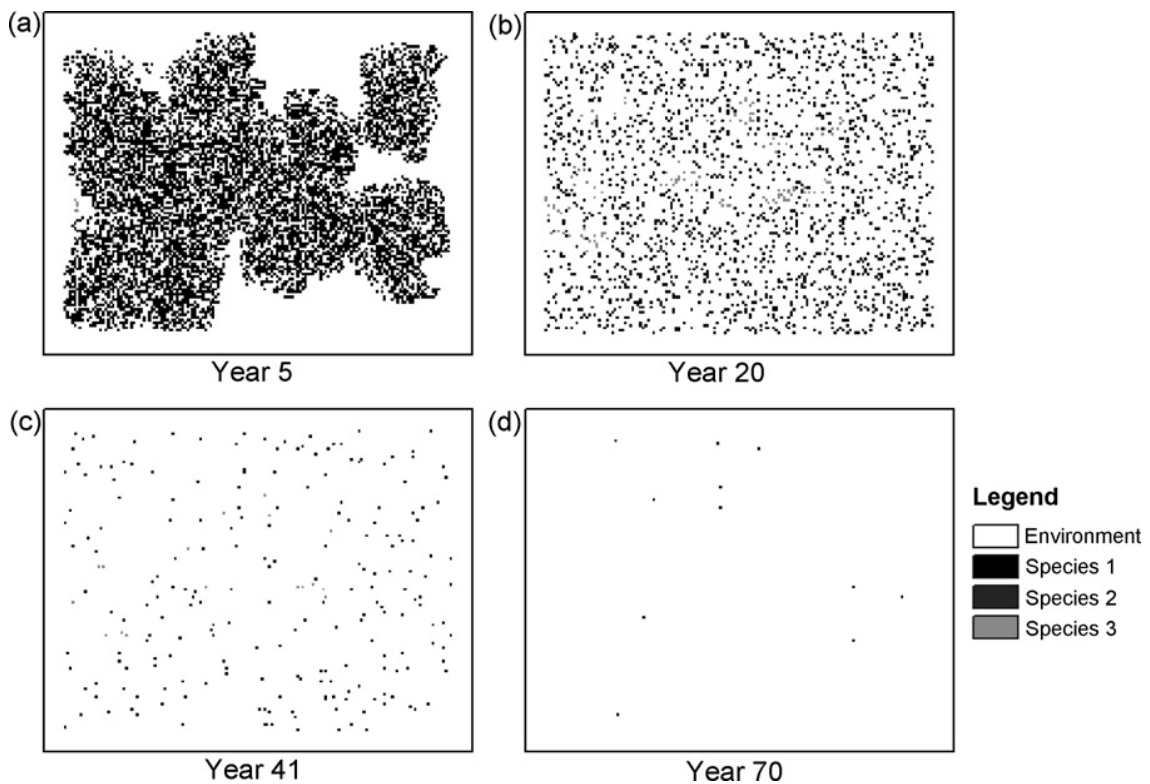

Fig. 7. The results of a 250 year seed dispersal simulation with environmental disturbance with the same initial configuration as Fig. 6a. (a) At age 5, species 1 colonized the majority of the landscape, which started to demonstrate a fragmented distribution. (b) At year 20, the three species still coexisted in a more fragmented landscape. (c) At year 41, species 2 became perished, with the two remaining species primarily distributed as individual trees instead of contiguous forest. (d) At year 70 , species 3 became extinct, earlier than species 1 , which persisted in the landscape until year 115 .

in Fig. 7b, especially given its dark gray color. It eventually perished the first in year 41 (Fig. 7c), similar to the case without habitat destruction in extinctive order. At this moment, the landscape was much more fragmented, and the remaining two species were primarily distributed as individual trees instead of contiguous forest. Species 3, the best competitor and the second best disperser became extinct in year 70 (Fig. 7d), earlier than species 1, demonstrating a different extinction sequence from the case without habitat destruction. Species 1, the poorest competitor and the best disperser, persisted in the landscape until year 115. At year 115, all species disappeared from the landscape as an ultimate outcome of habitat destruction. In this simulation, the order of extinction is primarily determined by seed dispersal ability, while the contribution of competitiveness of the species was greatly diminished due to the environment fragmentation imposed by habitat destruction.

It is observed that the degradation of the environment has alternated the effects of species competition. Meanwhile, the advantage of being a superior disperser is magnified. The progressive fragmentation by habitat destruction creates a substantial number of barriers in the landscape. Barriers greatly impede gravity dispersed seed propagation, preventing seeds from moving to a cell of a lower elevation. However, the effect on seed dispersal by wind is much less. Seeds carried by wind can cross the barriers when the width of the barrier is less than the maximum dispersal distance. Thus, the negative impact of habitat destruction is more than compensated for by reduced competition (Moilanen and Hanski, 1995).

\section{Conclusion and future study}

GIS spatial analysis tools made it possible to simulate the species colonization process by incorporating the behavioral characteristics of dispersal agents. By emphasizing the spatial process involved and the interaction between different species, forest dynamics in a landscape were modeled using a prototype of GIS based spatial explicit model of dispersal agent behavior (SEMODAR) model. As a more realistic CA model, SEMODAR simulated two of the major dispersal agents, wind and gravity agents. The simulation of these two agents laid the foundation for GIS based CA modeling of seed dispersal, and provides a good basis for adding more complex agents, such as animals and water, into the simulations. The powerful spatial analysis tools in GIS can provide a viable alternative for landscape ecologists to build more mechanism-oriented simulation models.

An artificial landscape was used in the experimental simulation to test the developed prototype and comparable results to previous studies in terms of extinction order of species in competitive environments with and without habitat destruction were obtained, which supports the original theoretical expectation. Species colonization ability is very important if an inferior competitor is to coexist with superior competitors in a competitive landscape. Environment fragmentation due to habitat destruction can have a biased impact on superior competitors if they are not efficient dispersers. Inferior competitors with fugitive migration ability, on the other hand, are favored by the reduced competition from superior competitors as a result of habitat destruction. The predictions about species competition that appear in previous studies are quite robust.

The findings of this study also reassure us of the critical role of seed dispersal ability in both the intact and disturbed environment. As one of the major colonization factors, seed dispersal ability is co-decided by maximum dispersal distance, maximum dispersal probability and dispersal mechanism. The 
type of dispersal agent is especially crucial for a species to survive in a fragmented landscape. Wind is a more efficient dispersal agent than gravity because it is less constrained by topography and barriers. With the same dispersal style, species with different maximum dispersal distances exhibit differing dispersal abilities.

Unlike LANDIS, the SEMODAR model is configured to work with landscape at micro spatial scales, where the dispersal mechanisms play predominant roles. Users of this model should set a scale that is appropriate for their research in question. The spatial resolution in the experimental simulation is set to be $30 \mathrm{~m}$, same as that of the digital elevation model used. The involvement of a coarse resolution may make some of the assumptions of the model weak. For example, it may not be realistic to assume that only one species can exist in each cell. Large cell size also prohibits the simulation of contagious seed dispersal, which is very common and important to species propagation, especially in the uphill direction. It is expected that if a finer cell size DEM is used, the simulation would be more accurate. The temporal resolution of the model can also be specified by users so that each modeling iteration can simulate the monthly, weekly or even daily dynamics of seed dispersal and establishment if necessary. In the simulation, each iteration stands for a period of 1 year due to the lack of detailed meteorology data. With the availability of environmental data at finer temporal resolution, the seasonal or perhaps monthly changes of the environment and their impact on the seed dispersal could be simulated. However, the refinement of either spatial or temporal resolution would exponentially increase the amount of data and time to simulate the process. Over the years, complex anemochory models have been proposed based on wind physics that take into consideration such variables as tree height, vertical wind velocity, and species-dependent seed terminal velocity (Andersen, 1991; Nathan et al., 2001; Waldron, 2002). In this prototype model, we simply did not include all these variables, due to the lack of the necessary data to account for them. With the availability of more detailed data in the future, this will be another area of our future improvements to the model.

For future study, we will also complete the simulation of two other agents for seed dispersal that are still currently under development: hydrochory and zoochory. Thus far, there have only been a few seed dispersal models that attempted to model these two dispersal agents (Waldron, 2002). For hydrochory dispersal, seeds are carried to their destinations by streams and rivers according to underlying the watershed. For zoochory dispersal, seeds are moved by animals along the routes to and from their main food and water sources. By including these two important agents we will be able to simulate seed dispersal patterns more accurately in future models.

The term "agent" employed in this paper is intended to describe a dispersal driven force that has the ability to impact on the environment, as opposed to the use of the "agent" concept used in the agent based modeling (ABM) approach, a very different type of "agent." Recently, becoming popular among spatial simulation models (Benenson and Torrens, 2004), ABM retain the concept of fixed automata in $\mathrm{CA}$ to describe environment status such as elevation, soil, temperature and other variables that can have dynamic values but static location. To better model the mobility of non-fixed automata, ABM introduced agent to represent an individual actor that is capable of moving through the action space and working towards a specific goal. Discrete mobile objects, such as human, insects and the seeds in our model are often modeled as agents in a typical ABM.

Like most seed dispersal models (Malanson and Cairns, 1997; He and Mladenoff, 1999; Martín-Herrero and CalviñoCancela, 2001), SEMODAR is fundamentally a raster-based CA model in construct. In such a pure CA model, the individuality of discrete seeds cannot be modeled directly. Instead, it is simulated indirectly through a tessellation representation, which is inefficient due to the inability to clearly track individual seeds in each cell. Bithell and Macmillan (2007) point out three inherent difficulties of strict raster-based CA modeling: fixed neighborhood geometry, cell-size-dependent spatial scale, and the restriction on number of occupants per cell. It was suggested that by introducing vector based simulation into the raster-based CA model, discrete seed objects can be emulated independently as active agents. Each environmental cell can then host multiple occupants. The spatial resolution of the model will not be completely restricted by raster cell size because vector data are spatial scale independent in nature. The simulation of dispersal behavior will no longer be subject to fixed neighborhood geometry. For example, it would not be necessary to use multiple moves of a $3 \times 3$ rectangle window to simulate gravity driven dispersal because a topographic profile can be directly generated between the current location and the seed source to determine the possibility of such a seed dispersal process. We have begun some preliminary work that aims to combine the stationarity of CA with the individuality and mobility of ABM in seed dispersal simulation (Qiu and Zou, 2006; Zou, 2005). GIS provides powerful capabilities to manipulate both vector and raster-based spatial data, offering the best flexibility to implement agent based simulation models for seed dispersal.

\section{References}

Andersen, M., 1991. Mechanistic models for the seed shadows of winddispersed plants. Am. Nat. 137, 476-497.

Batty, M., Couclelis, H., Eichen, M., 1997. Urban systems as cellular automata. Environ. Plan. B 24, 159-164.

Batty, M., Xie, Y., 1994. From cells to cities. Environ. Plan. B 21, 31-48.

Benenson, I., Torrens, P.M., 2004. Geosimulation: Automata-based Modelling of Urban Phenomena. John Wiley \& Sons., Hoboken, NJ.

Bithell, M., Macmillan, W.D., 2007. Escape from the cell: spatially explicit modelling with and without grids. Ecol. Model. 200, 59-78.

Clarke, K.C., Gaydos, L.J., 1998. Loose-coupling a cellular automaton model and GIS: long-term urban growth prediction for San Francisco and Washington/Baltimore. Int. J. Geogr. Inf. Sci. 12, 699-714.

Czárán, T., 1998. Spatiotemporal Models of Population and Community Dynamics. Chapman and Hall, New York.

Dytham, C., 1994. Habitat destruction and competitive coexistence: a cellular model. J. Anim. Ecol. 63, 490-491.

Dytham, C., 1995. Competitive coexistence and empty patches in spatially explicit metapopulation models. J. Anim. Ecol. 64, 145-146. 
Ellison, A.M., Bedford, B.L., 1995. Response of a wetland vascular plant community to disturbance: a simulation study. Ecol. Appl. 5, 109-123.

Gustafson, E.J., Shifley, S.R., Mladenoff, D.J., Nimerfro, K.K., He, H.S., 2000. Spatial simulation of forest succession and harvesting using LANDIS. Can. J. Forest Res. 30, 32-43.

Hanski, I., 1994. Spatial scale, patchiness and population dynamics on land [and discussion]. Philos. Trans.: Biol. Sci. 343, 19-25.

Hanson, J.S., Malanson, G.P., Armstrong, M.P., 1990. Landscape fragmentation and dispersal in a model of riparian forest dynamics. Ecol. Model. 49, 277-296.

He, H.S., Hao, Z., Mladenoff, D.J., Shao, G., Hu, Y., Chang, Y., 2005. Simulating forest ecosystem response to climate warming incorporating spatial effects in north-eastern China. J. Biogeogr. 32, 2043-2056.

He, H.S., Larsen, D.R., Mladenoff, D.J., 2002. Exploring component-based approaches in forest landscape modeling. Environ. Model. Software 17, 519-529.

He, H.S., Mladenoff, D.J., 1999. The effects of seed dispersal on the simulation of long-term forest landscape change. Ecosystems 2, 308-319.

He, H.S., Mladenoff, D.J., Boeder, J., 1999. An object-oriented forest landscape model and its representation of tree species. Ecol. Model. 119, 1-19.

Hodgson, M.E., Gaile, G.L., 1996. Characteristic mean and dispersion in surface orientations for a zone. Int. J. Geogr. Inf. Sci. 10, 817-830.

Li, X., Yeh, A.G.O., 2002. Neural-network-based cellular automata for simulating multiple land use changes using GIS. Int. J. Geogr. Inf. Sci. 16, 323-343.

Malanson, G.P., 1993. Riparian Landscapes. Cambridge University Press, Cambridge.

Malanson, G.P., Armstrong, M.P., 1996. Dispersal probability and forest diversity in a fragmented landscape. Ecol. Model. 87, 91-102.

Malanson, G.P., Cairns, D.M., 1997. Effects of dispersal, population delays, and forest fragmentation on tree migration rates. Plant Ecol. 131, 67-79.

Martín-Herrero, J., Calviño-Cancela, M., 2001. The design of a spatially explicit stochastic model for the simulation of oceanic seed dispersal. South Pacific J. Nat. Sci. 19, 42-48.

Mladenoff, D.J., He, H.S., 1999. Design and behavior of LANDIS, an objectoriented model of forest landscape disturbance and succession. In: Mladenoff, D.J., Baker, W.L. (Eds.), Advances in Spatial Modeling of Forest Landscape Change: Approaches and Applications. Cambridge University Press, Cambridge, UK, pp. 163-185.

Mladenoff, D.J., Host, G.E., Boeder, J., Crow, T.R., 1996. LANDIS: a spatial model of forest landscape disturbance, succession, and management. In: Goodchild, M.F., Steyaert, L.T., Parks, B.O. (Eds.), GIS and Environmental Modeling: Progress and Research Issues. GISWorld Books, Fort Collins, CO, pp. 175-180.

Moilanen, A., Hanski, I., 1995. Habitat destruction and coexistence of competitors in a spatially realistic metapopulation model. J. Anim. Ecol. 64, 141-144.

Murray, D.R., 1986. Seed Dispersal. Academic Press, New York.

Nathan, R., Safriel, U.N., Noy-Meir, I., 2001. Field validation and sensitivity analysis of a mechanistic model for tree seed dispersal by wind. Ecology 82, 374-388.

Nee, S., May, R.M., 1992. Dynamics of metapopulations: habitat destruction and competitive coexistence. J. Anim. Ecol. 61, 37-40.
Pacala, S.W., Canham, C.D., Saponara Jr., J., Silander, J.A., Kobe, R.K., Ribbens, E., 1996. Forest models defined by field measurements: estimation. Error Anal. Dyn. Ecol. Monogr. 66, 1-43.

Pacala, S.W., Canham, C.D., Silander Jr., J.A., 1993. Forest models defined by field measurements. I. The design of a northeastern forest simulator. Can. J. Forest Res. 23, 1980-1988.

Qiu, F., 1998. Simulation of seed dispersal in a competitive environment using a spatial behavioral model. In: Proceedings of the International Conference on Modeling Geographical and Environmental System with Geographical Information Systems (GIS) 2. pp. 645-651.

Qiu, F., Zou, Z. 2006, Cellular automata and agent-based modeling and simulation of forest distribution in a GIS environment, International Workshop of Forest Landscape modeling, June 20-24, 2006, Beijing, China. Abstract. http://www.landscape.ac.cn/workshop\%20home.htm.

Schurr, F.M., Bond, W.J., Midgley, G.F., Higgins, S.I., 2005. A mechanistic model for secondary seed dispersal by wind and its experimental validation. J. Ecol. 93, 1017-1028.

Shugart, H.H., West, D.C., 1977. Development of an appalachian deciduous forest succession model and its application to assessment of the impact of the chestnut blight. J. Environ. Manage. 5, 161-179.

Smith, T.M., Urban, D.L., 1988. Scale and resolution of forest structural pattern. Plant Ecol. 74, 143-150.

Takeyama, M., Couclelis, H., 1997. Map dynamics: integrating cellular automata and GIS through Geo-Algebra. Int. J. Geogr. Inf. Sci. 11, $73-91$.

Tilman, D., Lehman, C.L., Yin, C., 1997. Habitat destruction, dispersal, and deterministic extinction in competitive communities. Am. Nat. 149, 407435.

Tilman, D., May, R.M., Lehman, C.L., Nowak, M.A., 1994. Habitat destruction and the extinction debt. Nature 371, 65-66.

Tomlin, D., 1990. Geographic Information Systems and Cartographic Modeling. Prentice Hall, New York.

Urban, D.L., 1990. A Versatile Model to Simulate Forest Pattern: A User's Guide to ZELIG Version 1.0. Environmental Sciences, University of Virginia, Charlottesville.

van der Pijl, A., 1982. Principles of Dispersal in Higher Plants. Springer-Verlag, Berlin.

Wagner, D., 1997. Cellular automata and geographic information systems. Environ. Plan. B 24, 219-234.

Waldron, J.D., 2002. Modeling vegetation dispersal and diffusion: woody species responses to climate change and anthropogenic landscape alteration. Dissertation in Geography. Texas A\&M University, College Station, TX, p. 38.

White, R., Engelen, G., 1993. Cellular automata and fractal urban form: a cellular modeling approach to the evolution of urban land-use patterns. Environ. Plan. A 25, 1175-1199.

Wolfram, S., 1986. Theory and Applications of Cellular Automata. World Scientific, Singapore.

Wu, F., 1999. GIS-based simulation as an exploratory analysis for space-time processes. J. Geogr. Syst. 1, 199-218.

Zou, Z., 2005, Implementing agent-based modeling in a GIS environment. Thesis in GIS. University of Texas at Dallas, Richardson, TX, p. 32. 